\title{
INFLUENCE OF ELECTROMAGNETIC FIELD (1800 MHZ) ON LIPID PEROXIDATION IN BRAIN, BLOOD, LIVER AND KIDNEY IN RATS
}

\section{PAWEŁ BODERA ${ }^{1}$, WANDA STANKIEWICZ ${ }^{1}$, BOŻENA ANTKOWIAK², MAŁGORZATA PALUCH ${ }^{2}$, JAROSŁAW KIELISZEK ${ }^{1}$, JAROMIR SOBIECH ${ }^{1}$, and MARCIN NIEMCEWICZ ${ }^{3}$}

${ }^{1}$ Military Institute of Hygiene and Epidemiology, Warszawa, Poland

Department of Microwave Safety

${ }^{2}$ Military Institute of Hygiene and Epidemiology, Warszawa, Poland

Department of Pharmacology and Toxicology

${ }^{3}$ Military Institute of Hygiene and Epidemiology, Puławy, Poland

Biological Threat Identification and Countermeasure Center

\begin{abstract}
Objectives: The aim of this study is the evaluation of the influence of repeated ( 5 times for 15 min) exposure to electromagnetic field (EMF) of $1800 \mathrm{MHz}$ frequency on tissue lipid peroxidation (LPO) both in normal and inflammatory state, combined with analgesic treatment. Material and Methods: The concentration of malondialdehyde (MDA) as the end-product of the lipid peroxidation (LPO) was estimated in blood, liver, kidneys, and brain of Wistar rats, both healthy and those with complete Freund's adjuvant (CFA)-induced persistent paw inflammation. Results: The slightly elevated levels of the MDA in blood, kidney, and brain were observed among healthy rats in electromagnetic field (EMF)-exposed groups, treated with tramadol (TRAM/EMF and exposed to the EMF). The malondialdehyde remained at the same level in the liver in all investigated groups: the control group (CON), the exposed group (EMF), treated with tramadol (TRAM) as well as exposed to and treated with tramadol (TRAM/EMF). In the group of animals treated with the complete Freund's adjuvant (CFA) we also observed slightly increased values of the MDA in the case of the control group $(\mathrm{CON})$ and the exposed groups (EMF and TRAM/EMF). The MDA values concerning kidneys remained at the same levels in the control, exposed, and not-exposed group treated with tramadol. Results for healthy rats and animals with inflammation did not differ significantly. Conclusions: The electromagnetic field exposure (EMF), applied in the repeated manner together with opioid drug tramadol (TRAM), slightly enhanced lipid peroxidation level in brain, blood, and kidneys.
\end{abstract}

Key words:

Electromagnetic field, Lipid peroxidation, Malondialdehyde, Rats, Analgesics, Tramadol

\section{INTRODUCTION}

Radiofrequency electromagnetic fields (RF-EMFs; $1800 \mathrm{MHz}$ ) are generated by mobile telephones and their base stations and their utilization is increasing across the world. Low-energy RF-EMFs which may have biological effects, such as changes in oxidative metabolism after multiple exposures, have only been studied to a limited extent. When excess production of reactive oxygen

Received: March 15, 2014. Accepted: December 18, 2014.

Corresponding author: P. Bodera, Military Institute of Hygiene and Epidemiology, Department of Microwave Safety, Kozielska 4, 01-163 Warszawa, Poland (e-mail: pbodera@gmail.com). 
species (ROS) overwhelms the neutralizing capacity of cellular antioxidants, oxidative stress (OS) is established. Oxidative stress has been implicated as one of the main culprits in male infertility [1]. The pineal hormone, melatonin, which is reflected by the urinary metabolite 6-OMHS, is a known antioxidant, which protects against lipid peroxidation in the retina, brain, and liver cells. Mobile phone exposure leads to generation of free radicals and ROS levels in the testicular organs. Burch et al. [2] demonstrated reduction in 6-hydroxymelatonin sulfate (6-OHMS) level in urine among subjects using a cell phone for over $25 \mathrm{~min} /$ day.

Bortkiewicz et al. [3] examined possible effect of the exposure to the EMF emitted by a cellular phone on 6-hydroxymelatonin sulfate (6-OHMS) excretion, which reflects melatonin levels in blood. Each subject in the studied group was examined twice on a day without exposure and on a day with continuous exposure (60 min exposure arising from a cellular phone, frequency $900 \mathrm{MHz}$, pulsed with $217 \mathrm{~Hz}$, SAR $1.23 \mathrm{~W} / \mathrm{kg}$ ). Authors demonstrated that the EMF emitted by cellular phones has no noticeable influence on the melatonin level.

Animal studies have shown various examples of how mobile phones induce ROS in the eyes, brain, kidneys, and endometrial lining of uterus [4-7]. Apart from generation of the ROS, the electromagnetic field emitted from various devices (mobile phones and microwave ovens) may also alter antioxidant enzyme activity.

The activity of antioxidant enzymes such as superoxide dismutase and glutathione peroxidase in erythrocytes in humans exposed to the RF-EMF was presented by Moustafa et al. [8].

The activity of catalase, superoxide dismutase (SOD) and glutathione peroxidase (GSH-Px) is decreased by chronic exposure to the RF-EMF and thus reduces the total antioxidant capacity in different organs of the body [9].

We have continued our past efforts in this study to recognize the bioeffects of microwave radiation exposure $(1800 \mathrm{MHz})$ on human health due to excessive usage of mobile phones and the assumption that the various studies on possible adverse effects of this exposure on human health provided contradictory results.

In our previous studies we concluded that:

- High frequency electromagnetic fields of $1500 \mathrm{MHz}$ and $1800 \mathrm{MHz}$, when applied alone, did not influence pain perception threshold to thermal stimuli. It did, however, account for an unwanted effect - the diminishing analgesic action of tramadol [10].

- Electromagnetic fields exposure of $1800 \mathrm{MHz}$ frequency and $20 \mathrm{~V} / \mathrm{m}$ intensity, which is similar to cell phones, did not markedly influence the nociceptive threshold to thermal stimuli, although it did transiently decrease analgesic efficacy of tramadol in rats with complete Freund's adjuvant-induced paw inflammation [11].

In the last study [12] we recorded interesting findings for the groups of rats receiving tramadol and, concurrently, being exposed to an electromagnetic field. The antioxidant capacity of blood values, which was measured by the oxygen radical absorbance capacity (ORAC), in the group treated with tramadol (TRAM), were lower than in the case of other groups. However, in the groups treated with the inflammatory factor Complete Freund Adjuvant (CFA) - antioxidant capacity in the exposed group EMF/TRAM did not differ from the control group.

We observed a certain synergic mode of action between applied EMFs and administered tramadol in rats treated with the CFA. The antioxidant capacity of blood of healthy rats was higher than that of rats which received only tramadol, and were exposed to EMFs.

The aim of this study has been the evaluation of the influence of repeated (5 times) exposure to the EMF on lipid peroxidation (LPO) in blood, liver, kidneys, and brain of Wistar rats, both healthy and those with persistent paw inflammation (CFA), treated with tramadol.

We have used the malondialdehyde (MDA) to assess lipid oxidative damage. 
Tramadol hydrochloride $(( \pm)$ trans-2-[(dimethylamino) methyl]-1-(3-methoxyphenyl)-cyclohexanol) is a centrallyacting analgesic agent, which is widely used as effective analgesic for severe acute and chronic pain conditions, such as neuropathic, cancer and postoperative surgical pain.

\section{MATERIAL AND METHODS}

\section{Animals}

Experiments were performed on male Wistar rats weighing 220-250 g, purchased from the Center of Experimental Medicine (Medical University of Bialystok, Poland). Animals were housed in cages on a standard 12:12 h light/ dark cycle. Water and food had been available ad libitum until the rats were transported to the laboratory, approximately $1 \mathrm{~h}$ before experiments. Animals presenting any symptoms of illness were excluded from the study. All testing was performed between 9:00 a.m. and 4:00 p.m. and the animals were used only once.

\section{ETHICS}

The experimental protocol was approved by the IV Local Ethics Committee for Animal Experimentation.

\section{Drugs and chemicals}

The Complete Freund's Adjuvant (CFA; heat killed Mycobacterium tuberculosis suspended in paraffin oil, $1 \mathrm{mg} / \mathrm{ml}$ ) was purchased from Sigma-Aldrich.

Tramadol hydrochloride (Tramal ${ }^{\circledR}$, Grünenthal, Germany) was used in the form of injectable solution in aqua for injection, $20 \mathrm{mg} / \mathrm{kg}$ body mass, by intraperitoneal route.

\section{Experimental procedure}

In 1st part of our study, the experiments were performed on healthy rats, and on rats with persistent inflammatory state, induced by the CFA injection in the 2nd part of our study.

Persistent inflammatory state (CFA) was elicited $24 \mathrm{~h}$ before the EMF exposure and drug application.

\section{Study design}

Sixty-four male Wistar rats were randomly divided into 8 groups, 8 animals for each. Two control groups: unexposed healthy rats and rats with inflammation, and 6 investigated groups: healthy rats and rats with inflammation (CFA).

Two groups of healthy rats and 2 groups of rats with inflammation were exposed. Each animal was assigned to the particular group until the end of the experiment, thus the healthy rats were not then treated with the CFA (Figure 1).

The animals in each group were continuously exposed to microwave radiofrequency at $1800 \mathrm{MHz}$ (EMF) for $15 \mathrm{~min}, 5$ times (day after day). Animals were injected with $0.1 \mathrm{ml}$ volume of the CFA into the plantar surface of the left hind paw. Animals were placed in pairs in Plexiglas enclosures positioned centrally, $1 \mathrm{~m}$ away from the EMF source, and exposed to the far-field range of an antenna at $1800 \mathrm{MHz}$ with the additional modulation, and the value of effective electric field $20 \mathrm{~V} / \mathrm{m}$ and effective magnetic field value $0.05 \mathrm{~A} / \mathrm{m}$. The propagation vector of the incident wave was parallel to the long axis of the animal's body.

Rats were submitted to 5 periods of 15 min exposure or were sham-exposed $(\mathrm{CON})$ and sham-exposed treated with tramadol (TRAM) with no voltage applied to the field generator in control groups.

The control group (CON) in healthy rats, which was sham-exposed, was injected with aqua for injection in the $1 \mathrm{ml} / \mathrm{kg}$ volume and the CFA in rats with inflammation. Immediately before the EMF exposure (TRAM/EMF and TRAM groups in healthy and with inflammation), rats had been injected intraperitoneally with tramadol in the $20 \mathrm{mg} / \mathrm{kg}$ dose (TRAM) or aqua for injection (EMF). After the experiments, the rats were subjected to general anesthesia induced by intraperitoneal injection of ketamine (Bioketan $10 \mathrm{mg} / \mathrm{kg}$, Vetoquinol Biowet) and xylazine (Xylapan $10 \mathrm{mg} / \mathrm{kg}$, Vetoquinol Biowet) and blood samples were collected by incision of the jugular vein. 


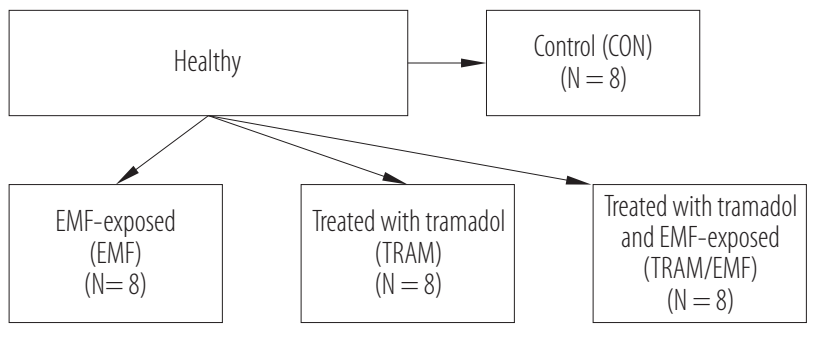

Fig. 1. Scheme of study design

\section{Specific Absorption Rate (SAR)}

We evaluated the Specific Absorption Rate (SAR) in rats which were exposed to a plane wave polarized electromagnetic field using a numerical model.

Finite-difference time-domain (FDTD) simulation with simplified homogeneous rat models was applied. The shapes of rat models were based on: magnetic resonance imaging (MRI) and computed tomography (CT) scans of the rat body, which were found in the Internet and literature. Rat pictures taken during the exposure and selected biometric data of exposed animals were compiled. The body weight of each rat model was approx. $231 \mathrm{~g}$ (the model volume was $217.9 \mathrm{~cm}^{3}$ and the assumed average density of rat body was $1.06 \mathrm{~g} / \mathrm{cm}^{3}$ ).

We assumed that the plane wave condition of the average permittivity and conductivity of the rat body are equal to average permittivity of a human body (equivalent to $2 / 3$ of muscle tissue). The electric properties of human muscle tissue were based on the calculation of the dielectric properties of body tissues in the frequency range of $10 \mathrm{~Hz}-100 \mathrm{GHz}$ [13].

The Specific Absorption Rate in rat models was calculated for the plain wave condition.

The electric field strength in terms of the RMS value was $20 \mathrm{~V} / \mathrm{m}$ and the frequency was equivalent to $1800 \mathrm{MHz}$. The rats were exposed in pairs inside the double box transparent plastic container.

The outer dimensions of the container were $218 \mathrm{~mm}$ in length and width, and the container height was $134 \mathrm{~mm}$. The container walls were $5 \mathrm{~mm}$ thick.

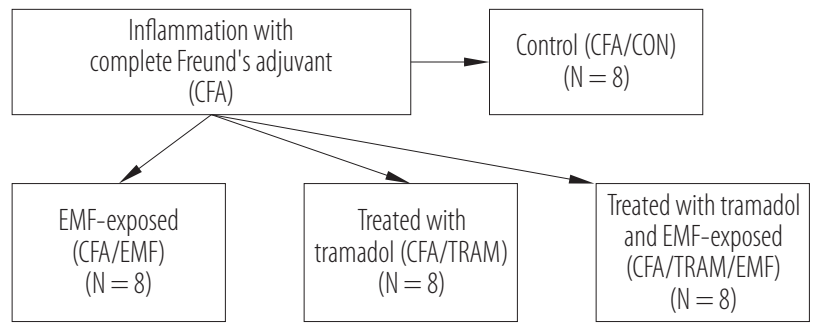

The rats were placed in pairs inside the container and each rat occupied 1/2 of the space of the container. During the exposure the rats were separated by a transparent plastic wall (5 $\mathrm{mm}$ in thickness) and could not move inside this space. Three scenarios of rat exposure were analyzed: - both rats are lying on the container floor and the wave vector $(\mathrm{k})$ is parallel to the long axes of animals,

- both rats are sitting on the container floor facing the antenna,

- both rats are climbing on the front wall of the container. The minimum value of the SAR was found in the 1st scenario, the maximum - for the scenario with rats climbing on the wall. The values of the SAR varied from 0.024 to $0.028 \mathrm{~W} / \mathrm{kg}$.

To validate the SAR calculations, the rat model dimensions were increased, and the volume of this new rat model was $314 \mathrm{~cm}^{3}$. The Specific Absorption Rate in this rat model was calculated at the plane wave condition when the electric field vector was parallel to the model long axis, the frequency was $1800 \mathrm{MHz}$ and the electric field strength in terms of the RMS value was $61.4 \mathrm{~V} / \mathrm{m}$ (equals to $1 \mathrm{~mW} / \mathrm{cm}^{2}$ at the plane wave condition). The results of this calculation correspond with the whole body SAR in medium rat models (model volume $320 \mathrm{~cm}^{3}$ ) reported for the group exposed to the above-mentioned conditions $[14,15]$.

\section{Electromagnetic field exposure system}

The electromagnetic field exposure system consisted of:

- Rohde \& Schwarz SMT06 Signal Generator $(5 \mathrm{kHz}-6 \mathrm{GHz})$, 
- TMD PTC6341 Amplifier (0.8-2 GHz, 250 W),

- Rohde \& Schwarz NRT-Z44 Directional Power Sensor (0.2-4 GHz, 0.003-120 W),

- EMCO 3115 Waveguide Horn Antenna (1-18 GHz).

The vertically-polarized antenna and exposure container with rats were placed inside the anechoic chamber.

The distance from the antenna aperture to the front wall of container equaled $1.2 \mathrm{~m}$.

The generator produced $1800 \mathrm{MHz}$ carrier wave with pulse modulation $(0.577 \mathrm{~ms}$ duration, repeating every $1.14 \mathrm{~ms}$ ). The method utilized by the GSM is a combination of time- and frequency-division multiple access (TDMA and FDMA). Frequency-division multiple access involves the division of the (maximum) $25 \mathrm{MHz}$ bandwidth by frequency into 124 carrier frequencies spaced $200 \mathrm{kHz}$ apart. In this study, 1 carrier frequency is assigned to the base station. That carrier frequency is then divided in time using a TDMA scheme. The fundamental unit of time in this TDMA scheme (a burst period) lasts for $15 / 26 \mathrm{~ms}$ (approximately $0.577 \mathrm{~ms}$ ). Eight burst periods are grouped into a TDMA frame (120/26 ms or approximately $4.615 \mathrm{~ms}$ ), which forms the basic unit of a logical channel. Therefore, the radio interface of carrier wave may be concurrently transmitted to 8 mobile subscribers at the same frequency channel.

An electric field strength in each fundamental unit of time is regulated automatically and depends, inter alia, on the distance between the base station and the subscriber that occupies this particular time slot.

In this study base station signal has been simplified and replaced with $0.577 \mathrm{~ms}$ pulse train with $50 \%$ of duty cycle. This is applicable to a situation when time slots are alternately assigned to subscribers that remain far-distant and nearby to a base station antenna.

The electric field RMS value was calculated at the distance $1.3 \mathrm{~m}$ away from the antenna aperture (in the center of exposure container). The electric field strength RMS value was $20 \mathrm{~V} / \mathrm{m}$.
The results of calculation were verified with Wandel \& Goltermann EMR-200 electric field meter with probe no. 9 (measurement range: $0.01-18 \mathrm{GHz}, 0.5-1000 \mathrm{~V} / \mathrm{m}$ ). The EMR-200 meter was calibrated by the Electromagnetic Field Standards and Metrology Laboratory, Technical University of Wroclaw.

\section{Lipid peroxidation}

Lipid peroxidation (LPO) was estimated by measuring the concentration of the colored complex formed by malondialdehyde (MDA; end-product of LPO during reaction) with externally-added thiobarbituric acid (TBA), according to Buege and Aust's method [16].

Portions of brain, liver, and kidneys $(250 \mathrm{mg})$ were homogenized in $1 \mathrm{ml}$ of ice-cold $0.15 \mathrm{M} \mathrm{KCl}$ using homogenizer OMNI TH. Then $4 \mathrm{ml}$ of $0.15 \mathrm{M} \mathrm{KCl}$ was added and all samples were centrifuged $\left(15 \mathrm{~min}, 2000 \times \mathrm{g}, 4^{\circ} \mathrm{C}\right)$. Obtained samples of supernatants $(1 \mathrm{ml})$ or plasma samples were diluted with $1 \mathrm{ml}$ of mixture consisting of $0.25 \mathrm{M} \mathrm{HCl}, 15 \%$ trichloroacetic acid and $0.375 \% \mathrm{TBA}$ and incubated $\left(15 \mathrm{~min}, 100^{\circ} \mathrm{C}\right)$. After cooling, all samples were centrifuged $\left(10 \mathrm{~min}, 4000 \times \mathrm{g}, 25^{\circ} \mathrm{C}\right)$. The TBA reactivity was assayed by measuring the absorbance of the supernatants at $532 \mathrm{~nm}$ at room temperature with the Lambda $35 \mathrm{UV} /$ vis spectrophotometer (Perkin Elmer). The MDA was calculated using the molar extinction coefficient $1.56 \times 105 \mathrm{M}^{-1} \times \mathrm{cm}^{-1}$.

\section{Statistical analysis}

The 1-way analysis of variance ANOVA for comparison within each group of rats (healthy and CFA-treated) and the 2-way ANOVA for comparison of both groups were applied. Significance between the groups was verified with Newman-Keuls post hoc test [17].

\section{RESULTS}

We observed slightly elevated levels of the MDA (expressed in terms of nmol/l) among healthy rats in 


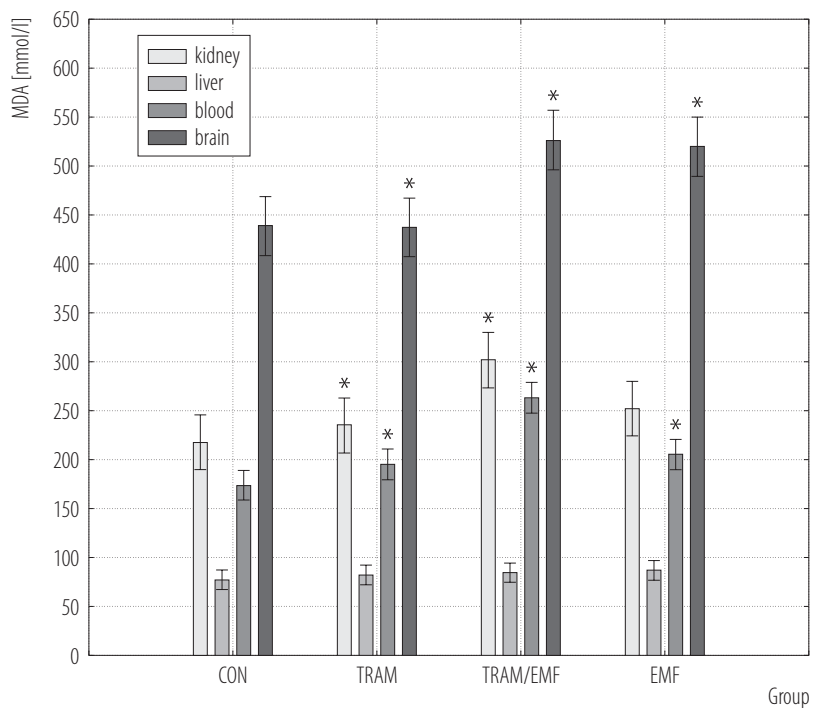

MDA - malondialdehyde; CON - the control group; TRAM - the tramadol treated group; TRAM/EMF - tramadol treated and the group exposed to the EMF; EMF - the group exposed to the EMF.

* Values significantly different ( $\mathrm{p}<0.05$ or less) from the corresponding control group.

Fig. 2. Lipid peroxidation expressed as malondialdehyde levels (nmol/l) among healthy rats

the EMF-exposed groups (TRAM/EMF and EMF), in blood, kidney, and brain in comparison with the control group (CON) (Figure 2). The malondialdehyde remained at the same level in the liver in all investigated groups (CON, EMF, TRAM, TRAM/EMF). In the CFA-treated animals we also observed slightly increased values of the MDA in the case of the control group (CON) and exposed groups (EMF and TRAM/ EMF). The MDA values concerning kidneys remained at the same levels in the case of the control, exposed and not-exposed group treated with tramadol (Figure 3).

Results for healthy rats and animals undergoing inflammation (CFA) did not differ significantly.

Our results were statistically significant in the following group in healthy rats: exposed (EMF) - for blood and brain values, exposed and treated with tramadol (TRAM/

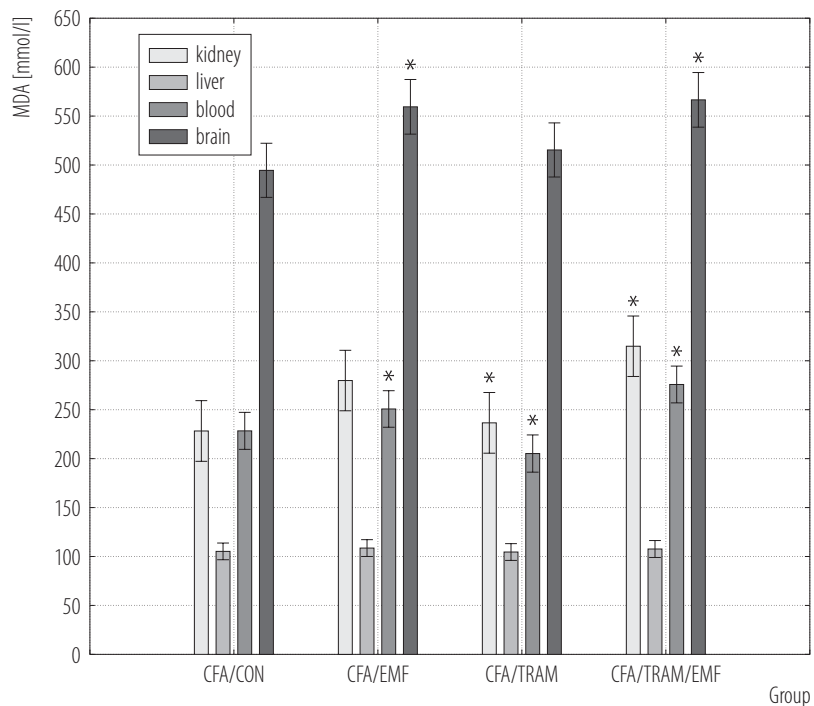

$\mathrm{CFA} / \mathrm{CON}$ - the complete Freund's adjuvant control group; CFA/EMF - the complete Freund's adjuvant exposed to the EMF group; CFA/TRAM - the complete Freund's adjuvant tramadol treated group; CFA/TRAM/EMF - the group exposed to the EMF and treated with complete Freund's adjuvant and tramadol.

* Values significantly different ( $\mathrm{p}<0.05$ or less) from the corresponding control group.

Fig. 3. Lipid peroxidation expressed as malondialdehyde levels (nmol/l) among rats with inflammation

EMF) - kidney, blood, and brain, and treated with tramadol without exposure (TRAM) - kidney, blood, and brain . In groups with inflammation there were the following values in the exposed group (CFA/EMF) - blood and brain, unexposed and treated with tramadol (CFA/TRAM) kidney, blood, and brain, and exposed and treated with tramadol (CFA/TRAM/EMF).

\section{DISCUSSION}

There is considerable apprehension worldwide since the use of mobile phones has expanded, along with the observation that the EMF radiation may have undesirable bioeffects.

Exposure to RF radiation generated by mobile phones was suggested as a trigger for a variety of neurological effects, including modification in the neuronal electrical 
activity changes in sleep pattern and disturbance in neurotransmitter release [18]. Although such a hypothesis may be controversial, the oxidative stress may be involved in the adverse effects elicited by RF-EMFs in the nervous system based on the increased evidence.

Ilhan et al. [19] reported oxidative damage in brain tissues of rats exposed to $900 \mathrm{MHz}$ signal for GSM (Global System for Mobile communications) for 7 days, which may support this assumption. They presented elevated levels of nitric oxide (NO), malondialdehyde (MDA), xanthine oxidase $(\mathrm{XO})$, as well as adenosine deaminase (ADA), and histopathological changes typical for brain injury, typically accompanied by oxidative stress, which may suggest a relationship with RF-EMFs exposure. Another study presented that the UMTS exposure (1950 MHz, 0.5 and $2 \mathrm{~W} / \mathrm{kg}$ ) did not induce oxidative stress in human lymphoblastoid cells, with the RF-EMF alone or in combination with ferrous ions, $\mathrm{FeSO}_{4}$ [20]. They measured the production of the ROS by flow cytometry. The 2 kinds of exposure (short 5-60 min or long $24 \mathrm{~h}$ ), which were carried out in a waveguide system, did not increase the spontaneous ROS formation under any of the investigated experimental conditions.

Zmyślony et al. [21] presented results of their study, based on the hypothesis that the $930 \mathrm{MHz}$ continuous wave $(\mathrm{CW})$ electromagnetic field may affect the reactive oxygen species (ROS) level in living cells. Rat lymphocytes were used in the experiments, where a part of them was treated with iron ions to induce oxidative processes.

Exposures to electromagnetic radiation (power density $5 \mathrm{~W} / \mathrm{m}^{2}$, theoretically calculated SAR $=1.5 \mathrm{~W} / \mathrm{kg}$ ) were performed. Intracellular ROS were measured by the fluorescent probe dichlorofluorescin diacetate (DCF-DA). The results show that acute (5 and $15 \mathrm{~min}$ ) exposure does not affect the number of produced ROS. Catalase, total antioxidative capacity (TAC), total oxidant status (TOS), and oxidative stress index were measured by Dasdag et al. [22], due to assessment of the effects of $900 \mathrm{MHz}$ during very long 10-month (2 h/day, 7 days/ week) exposure. They presented elevated levels of the TAC and catalase in case of the exposed group, while the TOS and oxidative stress index did not show any statistical difference between the exposed and the sham groups. $\mathrm{Xu}$ et al. [23] exposed primary cultured cortical neurons to pulsed RF-EMF electromagnetic fields (1800 MHz). They found that RF-EMF radiation induced a significant increase in the levels of 8-hydroxyguanine (8-OHdG), which is a common biomarker of DNA oxidative damage, in the mitochondria of neurons $24 \mathrm{~h}$ after the exposure. They also observed that the number of mtDNA copies and the levels of mitochondrial RNA (mtRNA) transcripts were reduced after the RF-EMF exposure.

The intensity of oxidative stress, cognitive impairment and inflammation in the brain of Fischer-344 rats exposed to the EMF radiation was evaluated by Megha et al. [24]. Rats were exposed to $1800 \mathrm{MHz}$ microwave radiation for 30 days ( $2 \mathrm{~h} /$ day).

Significant impairment in cognitive function and induction of oxidative stress in brain tissues of the EMF-exposed Male Fischer-344 rats was observed in comparison with the sham-exposed groups. They concluded that increased oxidative stress due to the EMF exposure may contribute to cognitive impairment and inflammation in the brain.

The lipid peroxidation in the brain tissue of pregnant and non-pregnant New Zealand White rabbits exposed to $1800 \mathrm{MHz}$ RF-EMF, resulting in oxidative DNA damage, was investigated by Guler et al. [25]. Rabbits were exposed to RF-EMF (1800 MHz GSM; $14 \mathrm{~V} / \mathrm{m}$ ) for $15 \mathrm{~min} /$ day during 7 days. The malondialdehyde (MDA) and 8-hydroxy-2'-deoxyguanosine (8-OHdG) levels of non-pregnant and pregnant EMF-exposed animals significantly increased with respect to control group ( $p<0.001)$.

The accumulated experience has led us to seek answers to the following questions: could some organs, like brain, liver, and kidneys be vulnerable to repeated EMF 
exposures, and could inflammation be an additional factor which may intensify such effects?

Bearing in mind the result of brain high metabolic rate, its diminished cellular turnover and its inefficient oxidant defense mechanism, the nervous system is particularly vulnerable to oxidative stress. The obtained results suggest that exposure to the EMF slightly increases tissue lipid peroxidation in some tissues, especially in brain and kidneys in adult healthy rats and rats with inflammation.

Administration of tramadol together with the EMF exposure enhanced lipid peroxidation in kidneys, blood, and brain, both in healthy rats and those with inflammation, which was similar to the results of our previous study carried out by another method.

In our previous study we stated that the antioxidant capacity of blood decreased in the exposed groups treated concurrently with tramadol and in this study we have concluded that the level of liver peroxidation increased, suggesting the higher activity of free radicals in studied tissues which, may lead to their damage.

\section{CONCLUSIONS}

It can be concluded that the increased risk of oxidative stress in various organs due to electromagnetic radiation should be taken into account. However, this risk burden is probably determined by the duration of exposure, SAR and also additional environmental factors.

\section{REFERENCES}

1. Agarwal A, Makker K, Sharma R. Clinical relevance of oxidative stress in male factor infertility: An update. Am J Reprod Immunol. 2008;59(1):2-11, http://dx.doi.org/10.1111/j.16000897.2007.00559.x.

2. Burch JB, Reif JS, Noonan CW, Ichinose T, Bachand AM, Koleber TL, et al. Melatonin metabolite excretion among cellular telephone users. Int J Radiat Biol. 2002;78(11): 1029-36, http://dx.doi.org/10.1080/09553000210166561.
3. Bortkiewicz A, Pilacik B, Gadzicka E, Szymczak W. The excretion of 6-hydroxymelatonin sulfate in healthy young men exposed to electromagnetic fields emitted by cellular phone - An experimental study. Neuro Endocrinol Lett. 2002;23 Suppl 1:88-91.

4. Oktem F, Ozguner F, Mollaoglu H, Koyu A, Uz E. Oxidative damage in the kidney induced by $900-\mathrm{MHz}-\mathrm{emit}-$ ted mobile phone: Protection by melatonin. Arch Med Res. 2005;36(4):350-5, http://dx.doi.org/10.1016/j.arcmed. 2005.03.021.

5. Balci M, Devrim E, Durak I. Effects of mobile phones on oxidant/antioxidant balance in cornea and lens of rats. Curr Eye Res. 2007;32(1):21-5, http://dx.doi.org/10. 1080/02713680601114948.

6. Meral I, Mert H, Mert N, Deger Y, Yoruk I, Yetkin A, et al. Effects of $900-\mathrm{MHz}$ electromagnetic field emitted from cellular phone on brain oxidative stress and some vitamin levels of guinea pigs. Brain Res. 2007;1169:120-4, http://dx.doi.org/10.1016/j.brainres.2007.07.015.

7. Guney M, Ozguner F, Oral B, Karahan N, Mungan T. $900 \mathrm{MHz}$ radiofrequency-induced histopathologic changes and oxidative stress in rat endometrium: Protection by vitamins E and C. Toxicol Ind Health. 2007;23(7): 411-20, http://dx.doi.org/10.1177/0748233707080906.

8. Moustafa YM, Moustafa RM, Belacy A, Abou-El-Ela SH, Ali FM. Effects of acute exposure to the radiofrequency fields of cellular phones on plasma lipid peroxide and antioxidase activities in human erythrocytes. J Pharm Biomed Anal. 2001;26(4):605-8, http://dx.doi.org/10.1016/S07317085(01)00492-7.

9. Kumar S, Kesari KK, Behari J. Evaluation of genotoxic effects in male Wistar rats following microwave exposure. Indian J Exp Biol. 2010;48(6):586-92.

10. Bodera P, Stankiewicz W, Antkowiak B, Paluch M, Kieliszek J, Sobiech J, et al. Suppressive effect of electromagnetic field on analgesic activity of tramadol in rats. Pol J Vet Sci. 2012;15(1):95-100, http://dx.doi.org/10.2478/ v10181-011-0119-3. 
11. Bodera P, Stankiewicz W, Antkowiak B, Paluch M, Kieliszek J, Sobiech J, et al. Suppressive effect of the pulsed electromagnetic field $1800 \mathrm{MHz}$ on analgesic action of tramadol in animal model of persistent inflammatory state. Centr Eur J Immunol. 2012;37(1):25-9.

12. Bodera P, Stankiewicz W, Zawada K, Antkowiak B, Paluch M, Kieliszek J, et al. Changes in antioxidant capacity of blood due to mutual action of electromagnetic field $(1800 \mathrm{MHz})$ and opioid drug (tramadol) in animal model of persistent inflammatory state. Pharmacol Rep. 2013;65(2):421-8, http://dx.doi.org/10.1016/S1734-1140(13)71017-X.

13. Dielectric properties of body tissues in the frequency range $10 \mathrm{~Hz}-100 \mathrm{GHz}$ [Internet]. Florence: Italian National Research Council, Institute for Applied Physics; 2014 [cited 2014 Jul 22]. Available from: http://niremf.ifac.cnr.it/ tissprop.

14. Durney CH, Massoudi H, Iskander MF. Radiofrequency radiation dosimetry handbook. 4th ed. San Antonio (TX): Armstrong Laboratory (AFMC); 1986.

15. Roach WP. Radio frequency radiation dosimetry handbook. 5th ed. Brooks City-Base: Air Force Research Laboratory; 2009.

16. Buege J, Aust S. Microsomal lipid peroxidation. Methods Enzymol. 1978;52:302-10, http://dx.doi.org/10.1016/S00766879(78)52032-6.

17. StatSoft, Inc. STATISTICA. Version 10. 2011 [cited 2011 Apr 26]. Available from: http://www.statsoft.com.

18. Hossmann KA, Hermann DM. Effects of electromagnetic radiation of mobile phones on the central nervous system. Bioelectromagnetics. 2003;24(1):49-62, http://dx.doi. org/10.1002/bem.10068.

19. Ilhan A, Gurel A, Armutcu F, Kamisli S, Iraz M, Akyol O, et al. Ginkgo biloba prevents mobile phone-induced oxidative stress in rat brain. Clin Chim Acta. 2004;340 (1-2):153-62, http://dx.doi.org/10.1016/j.cccn.2003.10.012.

20. Brescia F, Sarti M, Massa R, Calabrese ML, Sannino A, Scarfî MR. Reactive oxygen species formation is not enhanced by exposure to UMTS $1950 \mathrm{MHz}$ radiation and coexposure to ferrous ions in Jurkat cells. Bioelectromagnetics. 2009;30(7):525-35, http://dx.doi.org/10.1002/bem.20502.

21. Zmyślony M, Politanski P, Rajkowska E, Szymczak W, Jajte J. Acute exposure to $930 \mathrm{MHz} \mathrm{CW}$ electromagnetic radiation in vitro affects reactive oxygen species level in rat lymphocytes treated by iron ions. Bioelectromagnetics. 2004;25(5):324-8, http://dx.doi.org/10.1002/bem.10191.

22. Dasdag S, Akdag MZ, Kizil G, Kizil M, Cakir DU, Yokus B. Effect of $900 \mathrm{MHz}$ radio frequency radiation on beta amyloid protein, protein carbonyl, and malondialdehyde in the brain. Electromagn Biol Med. 2012;31(1):67-74, http://dx.doi.org/10.3109/15368378.2011.624654.

23. Xu S, Zhou Z, Zhang L, Yu Z, Zhang W, Wang Y, et al. Exposure to $1800 \mathrm{MHz}$ radiofrequency radiation induces oxidative damage to mitochondrial DNA in primary cultured neurons. Brain Res. 2010;1311:189-96, http://dx.doi. org/10.1016/j.brainres.2009.10.062.

24. Megha K, Deshmukh PS, Banerjee BD, Tripathi AK, Abegaonkar MP. Microwave radiation induced oxidative stress, cognitive impairment and inflammation in brain of Fischer rats. Indian J Exp Biol. 2012;50(12):889-96.

25. Guler G, Tomruk A, Ozgur E, Seyhan N. The effect of radiofrequency radiation on DNA and lipid damage in nonpregnant and pregnant rabbits and their newborns. Gen Physiol Biophys. 2010;29(1):59-66, http://dx.doi.org/10. 4149/gpb_2010_01_59.

This work is available in Open Access model and licensed under a Creative Commons Attribution-NonCommercial 3.0 Poland License - http://creativecommons.org/ licenses/by-nc/3.0/pl/deed.en. 